International Mathematical Forum, Vol. 9, 2014, no. 19, 917 - 921

HIKARI Ltd, www.m-hikari.com

http://dx.doi.org/10.12988/imf.2014.4490

\title{
Interval Images Recognition and Fuzzy Sets
}

\author{
G. Sh. Tsitsiashvili*, Yu. N. Kharchenko*, A. S. Losev* \\ and M. A. Osipova* \\ *Institute of Applied Mathematics Far Eastern Branch of the RAS \\ 690041 Vladivostok, Radio street 7, Russia
}

${ }^{* *}$ Far Eastern Federal University, 690950, Vladivostok, Sukhanov street 8, Russia

Copyright (c) 2014 G. Sh. Tsitsiashvili, Yu. N. Kharchenko, A. C. Losev and M. A. Osipova. This is an open access article distributed under the Creative Commons Attribution License, which permits unrestricted use, distribution, and reproduction in any medium, provided the original work is properly cited.

\begin{abstract}
In this paper a method of interval images recognition and its connection with fuzzy sets are described.
\end{abstract}

\section{Mathematics Subject Classification: $605 \mathrm{C} 80$}

Keywords: an interval images recognition, fuzzy sets, minimal and maximal segments

\section{Introduction}

In this paper a description of a method of interval images recognition [1] and its connection with fuzzy sets [2], [3] are considered. It was applied in a solution of some medical geography [4], meteorological [5] and etc. problems. But now this method needs mathematical development to smoothen boundaries of recognition intervals and to adopt it to different applied problems.

Main idea of [1] is a construction of multidimensional segments (rectangles) which divide a class of recognized objects into subclasses. Each subclass has its own maximal segment and minimal segment embedded in maximal one. Segments confronted to different subclasses do not intersect. 
In this paper for each subclass a membership function is defined. It equals: one in the minimal segment, zero out of the maximal segment and is redefined in an area between these segments using a similarity reason.

If a subject characterizes some catastrophical event (or its forerunner) then it may be predicted by sufficiently small meanings of a membership function because there is a large risk to miss this event. Vice versa to define limit parameters of some object it is better to increase meanings of a membership function.

Using geometrical reasons it is possible to calculate mean volume [6] of fuzzy set defined by multidimensional segments. A problem of mean volume calculation for general type fuzzy set origins from a data processing in geology and mining and is considered sufficiently detailed in papers on random sets [7, Chapters 5, 6]. Suggested definition of fuzzy set allows to adopt it to different conditions of applied problem and to avoid cumbersome procedure of a triangulation of convex set tightened on finite set of points. Last years in theory of fuzzy sets a necessity of such constructions is discussed in [8], [9].

\section{Multidimensional segments}

Assume that there is a finite set of $m$ - dimensional vectors $X_{i}=\left(x_{1, i}, \ldots, x_{m, i}\right)$, $1 \leq i \leq n$. Suppose that index set $\{1, \ldots, n\}$ is divided into two non intersected subsets $\{1, \ldots, p\},\{p+1, \ldots, n\}$. Denote $\mathcal{X}_{i}=\left\{x_{i, t}, p<t \leq n\right\}, 1 \leq i \leq m$. If real numbers $c, d$ coincide then an interval $(c, d)=\emptyset$.

Minimal segments. For any $i, j: 1 \leq i \leq m, 1 \leq j \leq p$, define numbers $k_{i, j} \leq r_{i, j}$ by equalities

$$
\begin{aligned}
& k_{i, j}=\min \left\{x_{i, t}: x_{i, t} \leq x_{i, j}, \mathcal{X}_{i} \bigcap\left(x_{i, t}, x_{i, j}\right)=\emptyset, 1 \leq t \leq p\right\}, \\
& r_{i, j}=\max \left\{x_{i, t}: x_{i, t} \geq x_{i, j}, \mathcal{X}_{i} \bigcap\left(x_{i, j}, x_{i, t}\right)=\emptyset, 1 \leq t \leq p\right\} .
\end{aligned}
$$

Then for any $i, j: 1 \leq i \leq m, 1 \underset{m}{\leq} j \leq p$, we have $\left.x_{i, j} \in \underset{i, j}{\left[k_{i, j}\right.}, r_{i}\right]$ and so for $j: 1 \leq j \leq p$, an inclusion $X_{j} \in \bigotimes_{i=1}^{m}\left[k_{i, j}, r_{i, j}\right]$ takes place. Here $\bigotimes_{i=1}^{m}\left[k_{i, j}, r_{i, j}\right]$ is direct product of segments $\left[k_{i, j}, r_{i, j}\right], 1 \leq i \leq m$.

Theorem 2.1 If indexes $j, j^{\prime}$ satisfy inequalities $1 \leq j \neq j^{\prime} \leq p$ then $m-$ dimensional segments $\bigotimes_{i=1}^{m}\left[k_{i, j}, r_{i, j}\right], \bigotimes_{i=1}^{m}\left[k_{i, j^{\prime}}, r_{i, j^{\prime}}\right]$ coincide or intersect in a set with zero Lebesgue measure.

Proof. If indexes $j, j^{\prime}$ satisfy inequalities $1 \leq j \neq j^{\prime} \leq p$ then for any $i: 1 \leq$ $i \leq m$, one dimensional segments $\left[k_{i, j}, r_{i, j}\right],\left[k_{i, j^{\prime}}, r_{i, j^{\prime}}\right]$ coincide or intersect 
in a set with zero Lebesgue measure. Indeed assume that for fixed $i, j \neq j^{\prime}$ between points $x_{i, j}, x_{i, j^{\prime}}$ on real axis there are not points from $\mathcal{X}_{i}$ then by a construction segments $\left[k_{i, j}, r_{i, j}\right],\left[k_{i, j^{\prime}}, r_{i, j^{\prime}}\right]$ coincide. Vice versa if between points $x_{i, j}, x_{i, j^{\prime}}$ on real axis there are points from $\mathcal{X}_{i}$ then by a construction segments $\left[k_{i, j}, r_{i, j}\right],\left[k_{i, j^{\prime}}, r_{i, j^{\prime}}\right]$ have no more than single joint point.

If one dimensional segments $\left[k_{i, j}, r_{i, j}\right],\left[k_{i, j^{\prime}}, r_{i, j^{\prime}}\right]$ for all $i: 1 \leq i \leq m$, coincide then their direct products coincide also. In opposite case there is at least single index $i$ for which these segments have no more than single joint point and so their direct products intersect in a set with zero Lebesgue measure. Theorem 2.1 is proved.

Remark 2.2 If there are $T$ classes of points in the space $E^{m}$ then using minimal $m$-dimensional segments it is possible to construct segments around points of all these classes. These segments coincide or have an intersection with zero Lebesgue measure but segments around points of different classes have an intersection with zero Lebesgue measure. This allows to apply such construction of minimal segments for a solution of the mapping problem which is one of the most important problems in geoinformatics and in data visualization.

Maximal segments. For any $i, j: 1 \leq i \leq m, 1 \leq j \leq p$, define

$$
K_{i, j}=\max \left\{x_{i, t} \in \mathcal{X}_{i}: x_{i, t} \leq k_{i, j}\right\}, R_{i, j}=\min \left\{x_{i, t} \in \mathcal{X}_{i}: x_{i, t} \geq r_{i, j}\right\} .
$$

Then for all $i, j: 1 \leq i \leq m, 1 \leq j \leq p$, we have $x_{i, j} \in\left[k_{i, j}, r_{i, j}\right] \subseteq\left[K_{i, j}, R_{i, j}\right]$ and so for any $j: 1 \leq j \leq p$, an inclusion $X_{j} \in \bigotimes_{i=1}^{m}\left[k_{i, j}, r_{i, j}\right] \subseteq \bigotimes_{i=1}^{m}\left[K_{i, j}, R_{i, j}\right]$ takes place.

Theorem 2.3 If indexes $j, j^{\prime}$ satisfy inequalities $1 \leq j \neq j^{\prime} \leq p$ then $m-$ dimensional segments $\bigotimes_{i=1}^{m}\left[k_{i}\left(x_{i}^{j}\right), r_{i}\left(x_{i}^{j}\right)\right], \bigotimes_{i=1}^{m}\left[k_{i}\left(x_{i}^{j^{\prime}}\right), r_{i}\left(x_{i}^{j^{\prime}}\right)\right]$ coincide or have an intersection with zero Lebesgue measure.

Proof of Theorem 2.3 almost word-for-word coincides with Theorem 2.1 proof.

\section{Construction of fuzzy segments}

Later assume that for each $i, 1 \leq i \leq m$, numbers $x_{i}^{j}, 1 \leq j \leq n$, are pairwise different. From Theorem 2.3 we have that the set of indexes $j$ : $1 \leq j \leq p$, is divided into equaivalence sublcasses. Elements of each subclass are embedded in same $m$ - dimensional segments $\bigotimes_{i=1}^{m}\left[K_{i, j}, R_{i, j}\right]$. Without a restriction of a generality assume that these classes are following indexes sets $\left\{1, \ldots, s_{1}\right\},\left\{s_{1}+1, \ldots, s_{2}\right\}, \ldots,\left\{s_{l-1}+1, \ldots, s_{l}=p\right\}$. Denote 
$B_{i, q}=K_{i, s_{q}}, b_{i, q}=k_{i, s_{q}}, a_{i, q}=r_{i, s_{q}}, A_{i, q}=r_{i, s_{q}}, 1 \leq i \leq m, 1 \leq q \leq l$. Define now fuzzy segment which denotes an inclusion of vector $X=\left(x_{1}, \ldots, x_{m}\right)$ in one of constructed $m$-dimensional segments. As these $m$-dimensional segments $\bigotimes_{i=1}^{m}\left[B_{i, q}, A_{i, q}\right]$ intersect in sets with zero Lebesque measure a membership function of this fuzzy set $\mu(X)=\sum_{q=1}^{l} \mu_{q}(X)$. Here for fixed $q, 1 \leq q \leq l$, function $\mu_{q}(X)$ is defined by conditions:

a) $X \in \mathcal{B}_{q}, \mathcal{B}_{q}=\otimes_{i=1}^{m}\left[b_{i, q}, a_{i, q}\right] \Longrightarrow \mu_{q}(X)=1$,

b) $X \notin \mathcal{A}_{q}, \mathcal{A}_{q}=\otimes_{i=1}^{m}\left[B_{i, q}, A_{i, q}\right] \Longrightarrow \mu_{q}(X)=0$,

c) assume that for $0 \leq \lambda \leq 1$ there is the inclusion $X \in \delta G_{\lambda}$ where $\delta G_{\lambda}$ is the boundary of the set $G_{\lambda}=\bigotimes_{i=1}^{m}\left[b_{i, q}+\lambda\left(B_{i, q}-b_{i, q}\right), a_{i, q}+\lambda\left(A_{i, q}-a_{i, q}\right)\right]$ then $\mu_{q}(X)=1-\lambda$, and so for $X \in \mathcal{A}_{q} \backslash \mathcal{B}_{q}=\bigcup_{0 \leq \lambda \leq 1} \delta G_{\lambda}$

$$
\mu_{q}(X)=1-\max _{1 \leq i \leq m} \max \left[\frac{x_{i}-a_{i, q}}{A_{i, q}-a_{i, q}}, \frac{x_{i}-b_{i, q}}{B_{i, q}-b_{i, q}}\right]
$$

It is obvious that for $m=1$ a graphic of the function $\mu_{q}\left(x_{1}\right)$ is a trapezium. Analogously with random sets [6] it is natural to define mean volume of fuzzy set $V$ defined by $m$-dimensional segments in the space $E^{m}$ by the equality $V=\int_{E^{m}} \mu(X) d X$. It is not difficult to check that the volume $V$ satisfies the equality: $V=\sum_{q=1}^{l} V_{q}, V_{q}=\sum_{q=1}^{l} \int_{E^{m}} \mu_{q}(X) d X$. Here from Fubini's Theorem

$$
\begin{gathered}
V_{q}=\int_{0}^{1} \prod_{i=1}^{m}\left(\left(a_{i, q}-b_{i, q}\right)(1-\lambda)+\lambda\left(A_{i, q}-B_{i, q}\right)\right) d \lambda=C_{q} \int_{0}^{1} D_{q}(\lambda) d \lambda, \quad \\
C_{q}=\prod_{i=1}^{m}\left(A_{i, q}-B_{i, q}-a_{i, q}+b_{i, q}\right), D_{q}(\lambda)=\prod_{i=1}^{m}\left(\lambda+\alpha_{i, q}\right), \alpha_{i, q}=\frac{a_{i, q}-b_{i, q}}{A_{i, q}-B_{i, q}-a_{i, q}+b_{i, q}} .
\end{gathered}
$$

Suppose that $\mathcal{K}_{1}=\{1, \ldots, m\}, \mathcal{K}_{2}$ is the set of all indexes pairs $\left(k_{1}, k_{2}\right), k_{1}, k_{2} \in$ $\mathcal{K}_{1}, k_{1}<k_{2}, \mathcal{K}_{3}$ is the set of all indexes three-tuples $\left(k_{1}, k_{2}, k_{3}\right), k_{1}, k_{2}, k_{3} \in$ $\mathcal{K}_{1}, k_{1}<k_{2}<k_{3} ; \ldots, \mathcal{K}_{m}=(1,2, \ldots, m)$ then

$$
\begin{gathered}
D_{q}(\lambda)=\lambda^{m}+\lambda^{m-1} \sum_{k_{1} \in \mathcal{K}_{1}} \alpha_{k_{1}, q}+\lambda^{m-2} \sum_{\left(k_{1}, k_{2}\right) \in \mathcal{K}_{2}} \alpha_{k_{1}, q} \alpha_{k_{2}, q}+\ldots \\
+\lambda^{m-3} \sum_{\left(k_{1}, k_{2}, k_{3}\right) \in \mathcal{K}_{3}} \alpha_{k_{1}, q} \alpha_{k_{2}, q} \alpha_{k_{3}, q}+\ldots+\alpha_{1, q} \ldots \alpha_{m, q}
\end{gathered}
$$


and so from the formula (1)

$$
\begin{aligned}
V= & \sum_{q=1}^{l} C_{q}\left[\frac{1}{m+1}+\frac{1}{m} \sum_{k_{1} \in \mathcal{K}_{1}} \alpha_{k_{1}, q}+\frac{1}{m-1} \sum_{\left(k_{1}, k_{2}\right) \in \mathcal{K}_{2}} \alpha_{k_{1}, q} \alpha_{k_{2}, q}+\right. \\
& \left.+\frac{1}{m-2} \sum_{\left(k_{1}, k_{2}, k_{3}\right) \in \mathcal{K}_{3}} \alpha_{k_{1}, q} \alpha_{k_{2}, q} \alpha_{k_{3}, q}+\ldots+\alpha_{1, q} \ldots \alpha_{m, q}\right] .
\end{aligned}
$$

\section{References}

[1] G.Sh. Tsitsiashvili, Image recognition by multidimensional intervals, Reliability: Theory and Applications, 8(2) (2013), 21-23.

[2] L.A. Zadeh, Fuzzy sets, Information and Control, 8(3) (1965), 338-353.

[3] A. Kaufmann, Introduction a la theorie des sous-ensembles flous, Masson, Paris, 1973.

[4] E.I. Bolotin, G.Sh. Tsitsiashvili, I.V. Golycheva, Some aspects and perspectives of factor prognosys for the tick-borne encephalitis spots on a base of multidimansional time series, Parasitology, 36(2) (2002), 89-95.

[5] T.A. Shatilina, G.Sh. Tsitsiashvili, T.V. Radchenkova, Experience of application of interval images recognition method for prognosys of ice-cover extremity of Tatar strait (Japanese Sea), Meteorology and Hydrology, 3 (2006), 65-72. (In Russian).

[6] H. E. Robbins, On the measure of a random set, Ann. Math. Statist., 3 (1944/45), 70-74/342-347.

[7] R.V. Ambartzumian, J. Mecke, D. Shtoyan, Introduction in Stochastic Geometry, Nauka, Moscow, 1989. (In Russian).

[8] D. Chakraborty, D. Ghosh, Analytical fuzzy plane geometry II, Fuzzy Sets and Systems, 243 (2014), 84-109.

[9] A.A. Efremov, New operations with fuzzy numbers and intervals, Dokladi TUSURa, 1(27) (2013), 95-99. (In Russian).

\section{Received: April 11, 2014}

